\title{
Tiempo de Respuestas y Experiencia de Usuario Estudio Experimental
}

\author{
Delvis Echeverria \\ GeneXus Consulting \\ Montevideo, Uruguay
}

\begin{abstract}
Resumen - La investigación está fundamentada por un estudio experimental, enfocado en la evaluación del comportamiento de los usuarios finales interactuando con tiempos de respuestas en aplicaciones reales. Se tomará como base el aporte de los autores Doherty y Thadani, investigadores de IBM relacionado con los tiempos de respuestas, y las investigaciones de Jacob Nielsen. Se llevarán a cabo un test con usuarios finales, y se expondrán optimizaciones a través de del uso de herramientas que contribuirán a mejorar los tiempos de respuestas de las aplicaciones web. Todo esto engloba una primera instancia de un proyecto que se está llevando a cabo en la empresa GeneXus Consulting, pretendiéndose lograr un punto de encuentro entre el rendimiento de las aplicaciones y la experiencia de usuario.
\end{abstract}

Palabras claves - tiempo de respuestas, experiencia de usuario.

\section{INTRODUCCIÓN}

En la actualidad vivimos en tiempos donde todo el mundo parece tener prisa, queremos todo "ya" y no deseamos perder ni tan solo un minuto. Lo mismo sucede cuando navegamos por internet, buscamos información y somos tan exigentes que tiene que ser buena y presentada rápidamente. La WWW se ha convertido en una importante y popular herramienta de búsqueda de información. Esta permite el acceso a casi todos los tipos de información - desde educación hasta entretenimiento [1].

Las aplicaciones Web desempeñan un papel muy relevante en toda actividad empresarial e intercambio de información. Estas representan para cualquier institución la carta de presentación hacia clientes potenciales. Pero no es suficiente con satisfacer las necesidades funcionales en una aplicación Web, la facilidad que experimenten los usuarios con éstas determinará en gran medida su éxito o fracaso. La usabilidad es una de las características de calidad más importantes para las aplicaciones Web, además de funcionalidad, seguridad, entre otras.

Algunos conceptos relacionados:

La usabilidad (dentro del campo del desarrollo web) es la disciplina que estudia la forma de diseñar sitios web para que los usuarios puedan interactuar con ellos de la forma más fácil, cómoda e intuitiva posible. [2]

El profesor Ricardo Baeza-Yates define a la disciplina como "un concepto que engloba a una serie de métricas y métodos que buscan hacer que un sistema sea fácil de usar y de aprender. Al hablar de sistema, la referencia se hace a cualquier dispositivo que tenga que ser operado por un usuario. En esta categoría caen los sitios web, aplicaciones de software, hardware, etc. [3]

La ISO/IEC 25000 la define como: "La usabilidad se refiere a la capacidad de un software de ser comprendido, aprendido, usado y ser atractivo para el usuario, en condiciones específicas de uso". [4]
La Experiencia del Usuario representa un cambio emergente del propio concepto de usabilidad, donde el objetivo no se limita a mejorar el rendimiento del usuario en la interacción - eficacia, eficiencia y facilidad de aprendizaje-, sino que se intenta resolver el problema estratégico de la utilidad del producto y el problema psicológico del placer y diversión de su uso. [5]

Arhippainen y Tahti definen la Experiencia del Usuario sencillamente como la experiencia que obtiene el usuario cuando interactúa con un producto en condiciones particulares. [6] Nielsen and Norman Group la definen como: "concepto integrador de todos los aspectos de la interacción entre el usuario final y la compañía, sus servicios y productos".

Como resultado de los conceptos estudiados en la presente investigación se puede definir la experiencia de usuario como la sensación, sentimiento, respuesta emocional, valoración satisfacción y comportamiento del usuario respecto a un producto, resultado del fenómeno de interacción con el producto. Es claro que si una página web tarda mucho tiempo en cargarse, el usuario se cansará y sin pensarlo dos veces cerrará la pestaña del navegador y no volverá a visitar más el sitio. Por tanto este factor, al cual no siempre se le da la debida importancia a la hora de desarrollar un producto de software, afecta el tráfico del sitio Web, el posicionamiento del mismo, y al usuario final. [7]

Un ingeniero de Google (Urs Holzle), recalca que: [8]:

- Un retraso de $400 \mathrm{~ms}$ produce un descenso del 0,44 el posicionamiento orgánico.

- El 80\% de los usuarios deja de ver un vídeo si este se para durante su reproducción.

- La media de carga de un sitio web en la actualidad es de 4,9 segundos.

Otros ejemplos relacionados [9]:

- Insideline: logró disminuir su tiempo de carga de 9 a 1,4 segundos. Su aumento en publicidad fue del $3 \%$ y sus páginas vistas aumentaron en un $17 \%$.

- Shospzilla: pasó de 7 segundos a 2. La publicidad creció un $112 \%$ y las páginas vistas un $25 \%$.

- Amazon: calculó que 0,1 segundos de retraso implican una pérdida del $1 \%$ en sus ingresos.

- Facebook: estimó que servir sus páginas 0,5 segundos más lento le provoca una caída de tráfico del $3 \%$ y que 1 segundo provoca una caída del $6 \%$.

- Google Maps: redujo un 30\% el tamaño de sus ficheros y el número de peticiones aumentó un 30\%.

- Yahoo!: calculó que 0,4 segundos de retraso le causan una caída entre el 5\% y el 9\% del tráfico.

El estudio del impacto del tiempo de respuesta en los productos de software no es un tema novedoso. En 1968 Robert B. Miller intentó resumir los trabajos de la psicología cognoscitiva hasta la fecha generando una lista de situaciones de interacción hombre-computadora y los tiempos de respuesta 
deseables en cada caso. Probablemente el aporte fundamental sea la idea de que cuando los tiempos de respuesta del sistema caen más allá de un límite, la interacción fluida desaparece y la eficiencia decae significativamente. [10]

Optimizar los tiempos de respuestas tiene un enorme impacto en la experiencia de los usuarios, navegar de manera fluida y rápida en una web hace que el usuario se sienta cómodo y se quede más tiempo. Además mejora el posicionamiento web, debido a que a los buscadores les gusta que los sitios web carguen rápido; anteriormente se mencionó el estudio de Urs Holzle relacionado con la velocidad, específicamente un ingeniero del buscador más usado en la actualidad, lo que quiere decir que el tiempo de respuesta es un factor importante en el algoritmo de estos buscadores.

\section{ESTUDIO EXPERIMENTAL}

Para el estudio experimental se tendrán en cuenta 10 aplicaciones web y estará estructurado en 4 etapas:

\section{- Medición y Análisis de los tiempos de respuesta.}

Se realizará la medición de los tiempos de respuestas referentes a las 10 aplicaciones web con características similares. Además se analizarán estos tiempos de respuestas y serán ubicados dentro del rango de tiempos de respuesta propuesto por Nielsen. [11]

- Test de usuario enfocado en tiempos de respuestas. Para el test con usuarios, estarán involucrados 5 usuarios finales de las aplicaciones web escogidas. Para este punto seguiremos las siguientes actividades:

1. Realizar la selección del Usuario.

2. Proponer lista de tareas.

3. Observar y escuchar la evolución del usuario.

4. Elaborar reporte.

- Experimento propuesto por Walter J. Doherty y Ahrvind J. Thadani

Se realizará una validación del experimento propuesto por estos investigadores de IBM.

\section{- Elementos de optimización.}

A través de algunas herramientas generaremos posibles optimizaciones aplicables a las 10 aplicaciones web escogidas.

\section{A. Medición y Análisis de tiempos de respuesta.}

Cercano a 0.1 segundos: Provee una sensación de control directo, en la que el usuario siente que está manipulando el sistema tal como lo haría con un objeto físico.

- Cercano a 1 segundo: El usuario percibe el retraso, pero aún siente control dentro del sitio. En las páginas de carga, este atraso es aceptable y esperable.

- Cercano a 10 segundos: El usuario pierde su atención, el flujo es interrumpido y no hay sensación de control. En este caso, las pantallas de carga y los mensajes ayudan a minimizar el abandono. La Figura 1 muestra los por cientos relacionados.

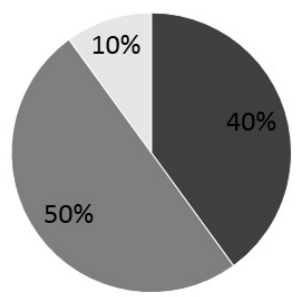

- Cercano a 0.1 a Cercano a 1.0 Cercano a 10.0

\section{B. Test de usuario enfocado en tiempos de respuestas.}

Para la seleccionar del usuario final, se tuvieron en cuenta usuarios finales relacionados con las aplicaciones web. Fueron escogidos 5 usuarios teniendo en cuenta lo propuesto por Nielsen [12], estos estuvieron interactuando con cada uno de las aplicaciones web. Como Objetivo en este Test de usuario se tuvo en cuenta: Medir la Tolerancia de los usuarios finales ante los tiempos de respuesta del sistema.

Fueron escogidos 5 usuarios finales. Luego de observar el comportamiento de los usuarios se generaron en el estudio experimental 3 indicadores relacionados con la interacción de los usuarios ante el aumento de los tiempos de respuestas:

- Abandono del sistema.

- Acciones innecesarias.

- Actualización del navegador (F5).

La distribución de los porcientos se muestra a continuación en la Figura 2.

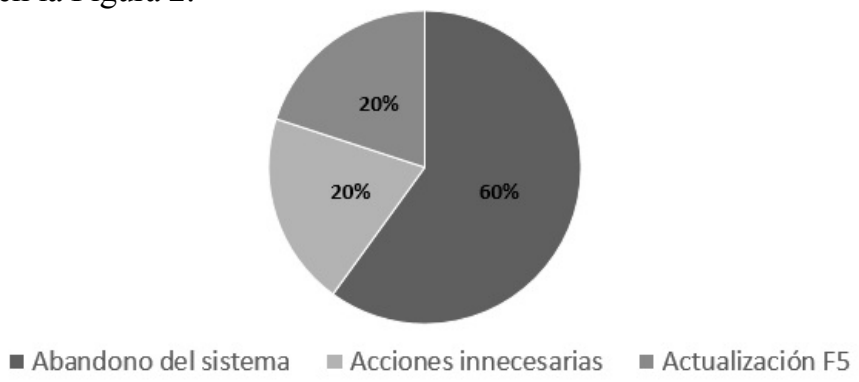

Fig. 2. Indicadores de comportamiento

Específicamente para el indicador Abandono del Sistema (ver Figura 3), se puede observar en la figura 3 que a los $35 \mathrm{~s}$, el $60 \%$ de los usuarios habían abandonado las aplicaciones.

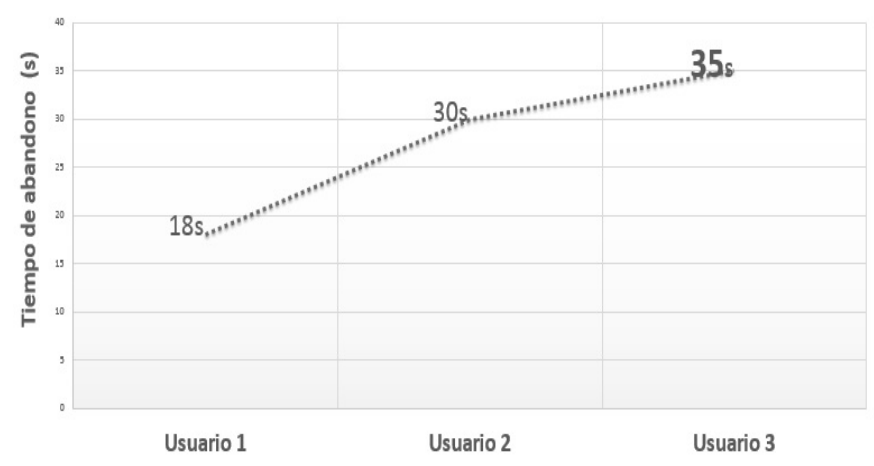

Fig. 3. Indicador "Abandono del Sistema"

\section{Experimento propuesto por Walter J. Doherty y Ahrvind J. Thadani.}

La propuesta de estos investigadores estuvo fundamentada en probar que el tiempo de respuesta del usuario disminuye a medida que disminuye el tiempo de respuesta del sistema (se explica gráficamente esta propuesta en la figura 4). Los investigadores describen que cada segundo de degradación en la respuesta del sistema conducía a una degradación similar al tiempo de respuesta del usuario. Relacionado estrechamente este fenómeno con la capacidad de atención de un individuo, las personas parecen tener una secuencia de acciones en mente, contenida en un búfer de memoria mental de corto plazo. El aumento del tiempo de respuesta del sistema pueden alterar los procesos de pensamiento, y esto puede dar lugar a tener que repensar la secuencia de acciones. [13]

Fig. 1. Por Ciento de Tiempos de Respuesta 


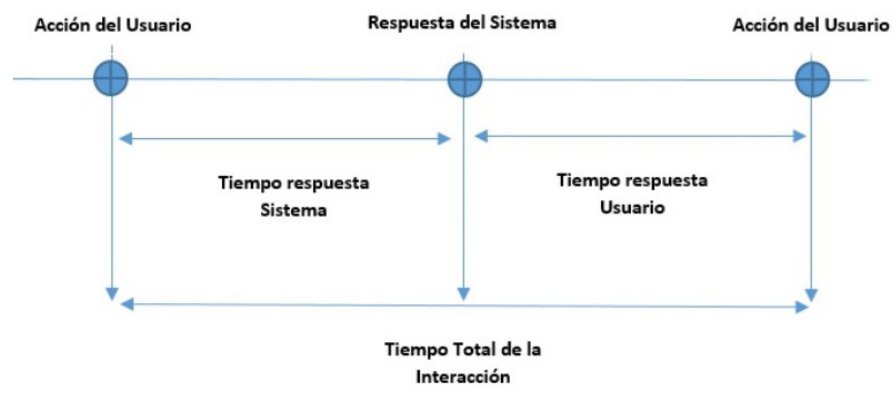

Fig. 4. Tiempos de respuestas y acciones del usuario

Seguido de la primera acción del usuario, el sistema emite una respuesta en un determinado tiempo (tiempo de respuesta sistema), y luego se espera por la próxima acción del usuario. El tiempo entre lo que respondió el sistema y la próxima acción del usuario, es el tiempo de respuesta del usuario, directamente proporcional con el tiempo de respuesta del sistema. Si aumentan los tiempos de respuestas sistema, aumentan los tiempos de respuestas de los usuarios.

Primeramente se calcularán los indicadores relacionados con los tiempos de respuestas para cada sistema web. Se pretende comprobar la relación que existe entre el tiempo de respuesta del sistema y el tiempo de respuesta del usuario. Para fundamentar y probar la propuesta de J. Doherty y Ahrvind J. Thadani, se calcularán primeramente los indicadores:

- Tiempos de respuesta del sistema (TRS)

- Tiempos de respuesta del usuario (TRU)

Para calcular los tiempos de respuesta se usaron las herramientas "Firebug" para calcular el tiempo de respuesta de las peticiones y "Steps Recorder" para medir los tiempos entre las acciones de los usuarios.

Tiempo Primera Acción: TPA

Tiempo Segunda Acción: TSA

Tiempo de Respuesta Sistema: TRS

Para calcular el TRU:

$$
T R U=(T S A-T P A)-T R S
$$

Por ejemplo:

$$
\begin{aligned}
\mathrm{TPA}=10 \mathrm{~s}, \mathrm{TSA}= & 13 \mathrm{~s}, \mathrm{TRS}=1.0 \mathrm{~s} \\
& \text { TRU }=(13 \mathrm{~s}-10 \mathrm{~s})-1.5 \mathrm{~s}=3 \mathrm{~s}-1.0 \mathrm{~s}=2.0 \mathrm{~s}
\end{aligned}
$$

Solo te tuvieron en cuenta los tiempos relacionados con el acceso a la página principal en las aplicaciones web. Se describen en la Tabla 1.

TABLA I. TIEMPOS DE RESPUESTAS

\begin{tabular}{|l|l|l|}
\hline \multicolumn{3}{|c|}{ Página Principal } \\
\hline Sitios Web & TRS & TRU \\
\hline Sistema 1 & 1.247 & 12.97 \\
\hline Sistema 2 & 1.912 & 7.648 \\
\hline Sistema 3 & 3.014 & 11.73 \\
\hline Sistema 4 & 1.009 & 5.915 \\
\hline Sistema 5 & 0.778 & 8.72 \\
\hline Sistema 6 & 0.228 & 9.407 \\
\hline Sistema 7 & 1.334 & 7.66 \\
\hline Sistema 8 & 2.610 & 6.222 \\
\hline Sistema 9 & 1.804 & 5.72 \\
\hline Sistema 10 & 4.506 & 19.77 \\
\hline
\end{tabular}

En un segundo escenario se aumentaron los tiempos de respuesta de los sistemas, para obtener nuevos indicadores para una posterior comparación. Se describe en la Tabla II.

TABLA II. TIEMPOS DE RESPUESTAS SEGUNDA INSTANCIA.

\begin{tabular}{|l|c|c|}
\hline \multicolumn{3}{|c|}{ Página Principal } \\
\hline Sitios Web & TRS & TRU \\
\hline Sistema 1 & 2.146 & 18.5 \\
\hline Sistema 2 & 3.887 & 18.25 \\
\hline Sistema 3 & 3.213 & 13.6 \\
\hline Sistema 4 & 1.439 & 11.7 \\
\hline Sistema 5 & 1.681 & 18.39 \\
\hline Sistema 6 & 1.020 & 11.41 \\
\hline Sistema 7 & 3.422 & 17.85 \\
\hline Sistema 8 & 4.222 & 24.35 \\
\hline Sistema 9 & 2.428 & 20.15 \\
\hline Sistema 10 & 7.982 & 26.23 \\
\hline
\end{tabular}

En la Tabla III se muestra la comparación de los tiempos de respuestas de los usuarios de ambas instancias.

TABLA III. COMPARACIÓN TIEMPOS RESPUESTAS USUARIOS

\begin{tabular}{|l|c|c|}
\hline \multicolumn{3}{|c|}{ Página Principal } \\
\hline Sitios Web & $\begin{array}{c}\text { TRU } \\
\text { 1ra Instancia }\end{array}$ & $\begin{array}{c}\text { TRU } \\
\text { 2da Instancia }\end{array}$ \\
\hline Sistema 1 & 2.146 & 18.5 \\
\hline Sistema 2 & 3.887 & 18.25 \\
\hline Sistema 3 & 3.213 & 13.6 \\
\hline Sistema 4 & 1.439 & 11.7 \\
\hline Sistema 5 & 1.681 & 18.39 \\
\hline Sistema 6 & 1.020 & 11.41 \\
\hline Sistema 7 & 3.422 & 17.85 \\
\hline Sistema 8 & 4.222 & 24.35 \\
\hline Sistema 9 & 2.428 & 20.15 \\
\hline Sistema 10 & 7.982 & 26.23 \\
\hline
\end{tabular}

Se puede constatar que los tiempos de respuestas del usuario se vieron afectados por el aumento del tiempo de respuesta del sistema. Todos aumentaron significativamente. Ver Figura 5. (Línea oscura primera instancia, línea clara segunda instancia)

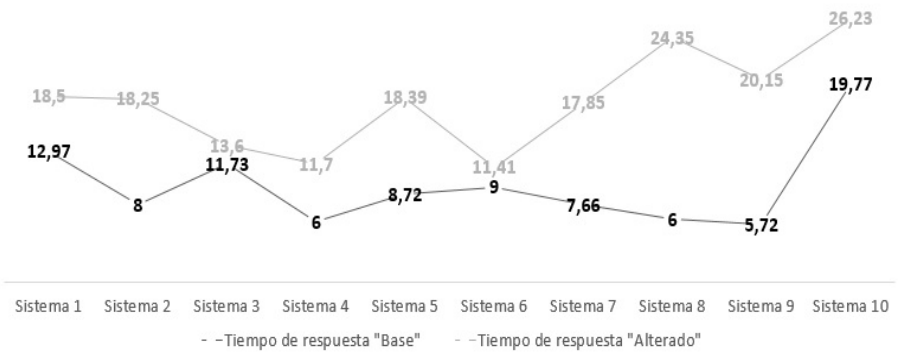

Fig. 5. Comparación tiempo respuesta usuario

\section{OPTIMIZACIÓN DE TIEMPOS DE RESPUESTA}

Las aplicaciones web seleccionadas para el estudio experimental propuesto estarán involucradas en el análisis de optimización que sigue a continuación.

Varias son las herramientas que nos permiten conocer qué debemos optimizar en nuestros sistemas web en cuanto a tiempos de respuestas. 
YSlow: analiza las páginas web y sugiere formas de mejorar el rendimiento, en base a un conjunto de 34 reglas de optimización con las que puedes mejorar el rendimiento de tu web y hacerla considerablemente más rápida. Las 34 normas se dividen en 6 categorías. Proporciona ccalificaciones basadas en tres conjuntos de reglas predefinidas a escoger por el usuario. Ofrece sugerencias para mejorar el rendimiento de la página. Resume los componentes de la página y Muestra estadísticas de la página. [14]

Pingdom: es una herramienta más sencilla que GTmetrix o Webpagetest, pero en contrapartida su análisis es muy rápido y la información muy clara. Muestra una lista de todos los elementos que se cargan al solicitar la página, y permite ordenarlos por tamaño, velocidad de carga entre otros. [15]

Google es una de las empresas que más importancia le da a los tiempos de respuesta de sus productos. No solo hace posible que los tiempos de respuesta de sus productos sean óptimos, además propone una herramienta que permite escanear a otros sistemas mostrándoles las posibles optimizaciones técnicas. PageSpeeds Insights es una herramienta que permite identificar maneras de hacer su sitio más rápido a través de reglas recomendadas por Google. Mide el rendimiento de las páginas para dispositivos móviles y para ordenadores. Obtiene la URL dos veces, una vez con un agente de usuario para móviles, y otra con un agente de usuario para ordenadores. La puntuación de PageSpeed va de 0 a 100 puntos. Cuanta más alta sea la puntuación, mejor. Por ejemplo, una puntuación de 85 indicaría que el rendimiento de la página es bueno, por lo que es posible que cambie la puntuación a medida que añadimos nuevas reglas o mejoremos nuestro sistema de análisis. [16]

Mostraremos para esta propuesta solamente las reglas afectadas usando PageSpeeds Insights.

- Optimizar imágenes

- Especificar caché de navegador

- Habilitar compresión

- Eliminar el JavaScript

- Minificar JavaScript

- Minificar CSS

- Minificar HTML

- Evita los re-direccionamientos a páginas de destino

- Prioriza el contenido visible

- Reducir el tiempo de respuesta del servidor

Los 10 sistemas web evaluados fueron analizados con PageSpeeds Insights, las principales reglas afectadas fueron "Optimizar Imágenes" con un 70\%, 7 de cada 10 aplicaciones web incurrieron en esta regla, "Habilitar compresión", $60 \%, 6$ de cada 10 aplicaciones web estuvieron afectadas por esta regla. Las restantes reglas se muestran en la Figura 6.

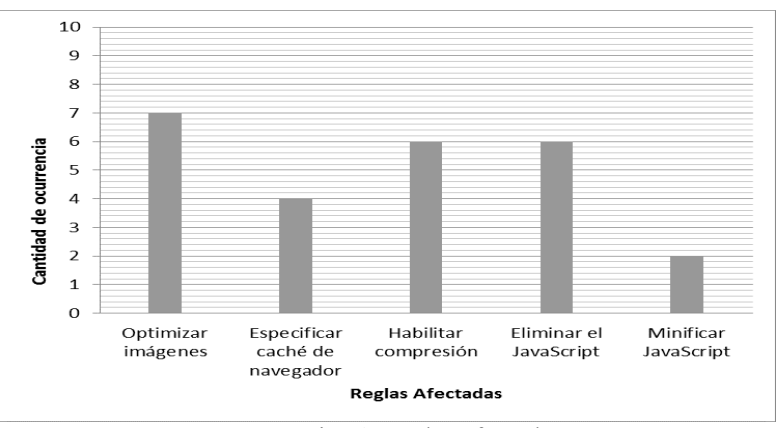

Fig. 6. Reglas afectadas.

\section{CONCLUSIONES}

De los 10 sistemas evaluados el $70 \%$ de los tiempos de respuesta se encuentran entre $1.0 \mathrm{~s}-2.0 \mathrm{~s}$, el $10 \%$ por debajo de 1.0 , el $20 \%$ se encuentran entre $3.0 \mathrm{~s}-10 \mathrm{~s}$.

Se comprobó que los tiempos de respuestas de los usuarios aumentan a medida que aumenta el tiempo de respuesta del sistema.

Se identificaron 3 indicadores de comportamiento de usuarios finales. El más significativo "Abandono del sistema" representó el $60 \%$.

Principales reglas de optimización afectadas son: Optimizar Imágenes, eliminar el JavaScript, habilitar compresión, especificar caché de navegador.

\section{REFERENCIAS}

[1] Selvidge, P. How long is too long for a website to load? http://psychology.wichita.edu/surl/usabilitynews/1s/time_delay. $\mathrm{htm}$

[2] Hassan, Y. Introducción a la Usabilidad. http://www.nosolousa bilidad.com/articulos/introduccion_usabilidad.htm

[3] Baeza, R. Ubicuidad y Usabilidad en la Web. http://www. dcc.uchile.cl/\%7Erbaeza/inf/usabilidad.html

[4] ISO/IEC 25010.

[5] D'Hertefelt, S. Emerging and future usability challenges: designing user experiences and user communities. http://www.i nteractionarchitect.com/future/vision20000202shd.htm

[6] Arhippainen, L., Tähti, M, Empirical Evaluation of User Experience in Two Adaptative Mobile Application Prototypes.

[7] Hassan, Y. Martín, F. La Experiencia del Usuario. http://www.nosolousabilidad.com/articulos/experiencia_del_usu ario.htm

[8] Urs H. 2012. The Google gospel of-speed.

[9] Anonimo. 2013, Cómo medir y mejorar la velocidad de carga de tu página web. http://www.ipixelestudio.com/medir-mejorarvelocidad-carga-pagina-web.html

[10] Mordecki, D. Miro y entiendo. Guía práctica de Usabilidad web. Capítulo 7, pag 207-2018.

[11] Jakob, N. 1993. Response Times: The 3 Important Limits https://www.nngroup.com/articles/response-times-3-importantlimits/

[12] Jakob, N. 2000. Why You Only Need to Test with 5 Users https://www.nngroup.com/articles/why-you-only-need-to-testwith-5-users/

[13] Elliott, J. 1982. The Economic Value of Rapid Response Time. http://www.vm.ibm.com/devpages/jelliott/evrrt.html

[14] YSlow. Página Oficial en Español. http://yslow.es/

[15] Pingdom. Página Oficial en Ingles https://www.pingdom.com

[16] PageSpeed Insights. https://developers.google.com/speed/docs/ insights/about

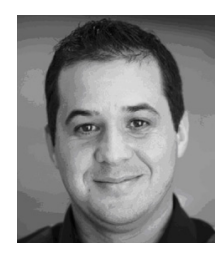

Delvis Echeverría. Graduado de Ingeniero Informático y Máster en Calidad de Software por la Universidad de las Ciencias Informáticas (UCI) en La Habana. Cuenta con más de 8 años en el área de QA y Testing en Cuba y Uruguay. Certificado ISTQB CTFL. Ha publicado varios artículos en revistas y congresos científicos. Actualmente se desempeña en el área de Performance Testing en GeneXus Consulting. 